\title{
High alpha-fetoprotein levels after liver transplantation in hepatoblastoma: Does it matter?
}

\author{
Benjamin Hetzer ${ }^{1}$, Rupert Oberhuber ${ }^{1}$, Andreas Entenmann ${ }^{1}$, Stefan Schneeberger ${ }^{1}$, and \\ Roman Crazzolara ${ }^{1}$ \\ ${ }^{1}$ Medical University of Innsbruck
}

November 28, 2020

\section{High alpha-fetoprotein levels after liver transplantation in hepatoblastoma: Does it matter?}

Hetzer Benjamin, M.D. ${ }^{1}$, Oberhuber Rupert, M.D. ${ }^{2}$, Entenmann Andreas, M.D. ${ }^{1}$, Schneeberger Stefan, M.D. ${ }^{2}$, Crazzolara Roman, M.D. ${ }^{1, *}$

${ }^{1}$ Department of Pediatrics I, Medical University of Innsbruck, Innsbruck, Austria

${ }^{2}$ Department of Visceral, Transplant and Thoracic Surgery, Medical University of Innsbruck, Innsbruck, Austria

${ }^{*}$ Correspondence to:

Roman Crazzolara, M.D., Department of Pediatrics, Anichstrasse 35, 6020 Innsbruck, Austria; Tel.: 0043512-504-23600; Fax: 0043-512-504-24934; Email: roman.crazzolara@i-med.ac.at

Hetzer Benjamin, M.D.: participated in research design and in the writing of the paper

Oberhuber Rupert, M.D.: participated in the performance of the research

Entenmann Andreas, M.D.: participated in research design

Schneeberger Stefan, M.D.: participated in the performance of the research

Crazzolara Roman, M.D.: participated in research design, in data analysis and in the writing of the paper

The authors declare no conflicts of interest.

The authors received no specific funding for this work.

This study was exempt from approval from the Ethics Committee of the Medical University of Innsbruck. Written informed consent was obtained from the caregivers of the patient for publication of this letter.

Dear Editor:

Serum alpha-fetoprotein (sAFP) is the main biomarker available for the management of unresectable hepatoblastoma undergoing liver transplantation in children(1). Although a rapid decline is indicative for appropriate response to neoadjuvant chemotherapy, the interval at which sAFP should be monitored after transplant and the exact cut-off value have not been fully determined(2). The questions are important, since patients with extended disease have high risk of tumor recurrence and in case sAFP is rising, elaborated imaging modalities are promptly necessitated.

We report a case of a 9-months-old infant with prenatally known Beckwith-Wiedemann syndrome and diagnosed with pretext IV hepatoblastoma at the age of 5 months. Size of hepatoblastoma was 10 x 8 
$\mathrm{cm}$ in diameter in the right hepatic lobe with additional multiple small lesions in both lobes of the liver. The sAFP concentration was $1048193 \mu \mathrm{g} / \mathrm{l}$ at the start of chemotherapy. After three courses of cisplatin and doxorubicin sAFP values dropped to $902 \mu \mathrm{g} / \mathrm{l}$. As complete tumor resection could not be achieved with partial hepatectomy, primary transplantation was performed at the age of 7 months. The patient received a full-size liver from a 2-months-old child after neurologic determination of death. Seven days after transplantation the sAFP level was $169 \mu \mathrm{g} / \mathrm{l}$ and within normal levels (at that time the patient showed extensive protein loss of $76 \mathrm{~g} / 24$ hours due to ascites). Following the institute post-transplant protocol, measurement of sAFP showed a dramatic increase to $2644 \mu \mathrm{g} / \mathrm{l}$ at day 27 . Other liver tests at that time were unremarkable (AST, ALT, GGT and bilirubine in normal range). As recurrence was suspected, immediate imaging diagnostics, including abdominal ultrasound, thoracic CT and full body MRI under intubation anesthesia were performed. Fortunately, no signs of tumor relapse were detected, sAFP levels dropped to normal values within the following weeks and no post-transplant chemotherapy was required (Figure 1). The patient could be discharged from hospital at 8 weeks after transplant and is in sustained remission at the last follow up after one year.

Taken together, this case illustrates that sAFP concentration in a child after liver transplant for hepatoblastoma can be highly elevated at week 4 after transplant, but caution is advised in using this biomarker as a screening tool for tumor recurrence. Firstly, fluctuating levels of sAFP can reflect acute liver failure (ALF), which is limited to the first week post-transplant and was not diagnosed in the current case(3). Furthermore, elevated sAFP levels can be associated with drugs causing ALF or flare-ups of viral hepatitis(3), which were negative in the current case. However, re-evaluation of organ circumstances revealed that the liver was obtained from a 2-months-old infant, in which elevated physiologic sAFP levels may be reflective of the young donor (Figure 1)(4).

This report demonstrates that sAFP levels can fluctuate after liver transplant and caution is needed in performing stressful investigations and in planning post transplantation chemotherapy. Future guidelines are required to determine the exact time points of sAFP analysis after liver transplant and to clarify if donor sAFP level should be collected as biomarker for assessing recurrence.

\section{Reference list}

1. Blohm ME, Vesterling-Horner D, Calaminus G, Gobel U. Alpha 1-fetoprotein (AFP) reference values in infants up to 2 years of age. Pediatr Hematol Oncol. 1998;15(2):135-42.

2. Trevisani F, Garuti F, Neri A. Alpha-fetoprotein for Diagnosis, Prognosis, and Transplant Selection. Semin Liver Dis. 2019;39(2):163-77.

3. Schiodt FV, Ostapowicz G, Murray N, Satyanarana R, Zaman A, Munoz S, et al. Alpha-fetoprotein and prognosis in acute liver failure. Liver Transpl. 2006;12(12):1776-81.

4. Duffy KA, Cohen JL, Elci OU, Kalish JM. Development of the Serum alpha-Fetoprotein Reference Range in Patients with Beckwith-Wiedemann Spectrum. J Pediatr. 2019;212:195-200 e2.

\section{Figure legend}

Figure 1: Development of serum alpha-fetoprotein (sAFP) levels in a 9-month-old child with hepatoblastoma after full- size liver transplantation from a 2-months-old donor (black graph) compared with estimated median physiologic sAFP levels in an 2-months-old preterm (dotted graph) or average 2-months-old child with Beckwith-Wiedemann syndrome (dashed graph)(4). 
Figure 1

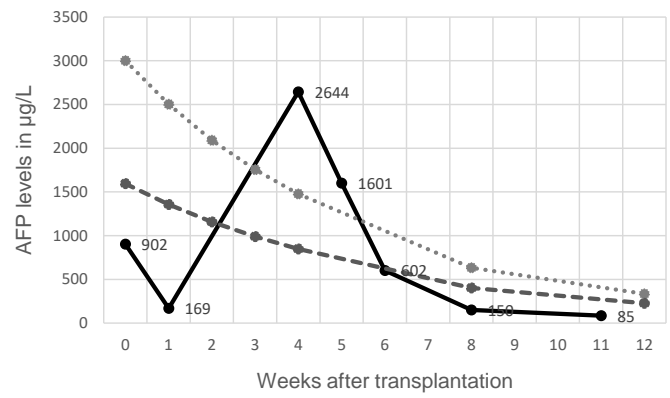

